\title{
Insensitivity of the Stationary Distribution of State Probabilities in an Open Network with Non-Active Customers
}

\author{
Yu. S. Kruk* and Yu. E. Dudovskaya** \\ ${ }^{*}$ Belarusian National Technical University, Minsk, Belarus \\ ** Francisk Skorina Gomel State University, Gomel, Belarus \\ e-mail: juls1982@list.ru,dudovskaya@gmail.com
}

Received December 7, 2014

\begin{abstract}
We study the stationary operation of an open queueing network with non-active customers and informational signals. The amount of work to service a claim is a random value with an arbitrary distribution. The stationary distribution of network state probabilities has a multiplicative form and is insensitive with respect to the functional form of the distribution of the work needed to service a claim.
\end{abstract}

DOI: $10.1134 / \mathrm{S} 0005117915120061$

\section{INTRODUCTION}

At present, the studies of reliability of servicing systems in the theory of queueing networks become more and more relevant. However, it is not only the servicing system that can stop working, the claims arriving in the system also may lose their qualitative characteristics.

From the point of view of reliability of incoming claims, queueing networks with non-active customers are especially interesting. Claims in such networks fall into two classes: some can be serviced by nodes while others are temporarily inactive and are not serviced, accumulating into queues at the nodes. The incoming flows of informational signals let claims change their state from inactive to the state where they can be serviced and vice versa. In most cases, researchers are interested in characteristics of stationary operation for such networks, in particular, the form of the stationary distribution of state probabilities.

Inactive claims can be interpreted as claims with some defect that makes them unfit for servicing. Indeed, in data transmission in informational and telecommunicational networks there may arise a situation when the transmitted claim becomes unfit for servicing as a result of some kind of fault in the process of its transmission. Thus, studies of networks with temporarily non-active claims present practical interest as well.

The work [1] considers stationary operation of an open network with non-active customers and studies the stationary distribution of state probabilities under the assumption that the servicing duration of the claims have exponential distribution. The works $[2,3]$ study the stationary operation of networks which generalize the model from [1] to the cases of the circulation of claims and signals of different kinds and flows of non-active claims arriving in the network.

A classical Jackson open queueing network has been studied in [4] under the assumption that a claim's servicing duration has exponential distribution, but this assumption seldom holds in practice. Indeed, in practice the distribution law of a claim's servicing duration is often far from exponential, so studies of queueing networks with arbitrary distribution functions of the servicing time represent an important problem in queueing theory and has been attracting more and more 
attention of the researchers [5-9]. The works $[8,9]$ are devoted to the studies of insensitivity for the stationary distribution of state probabilities in closed and open queueing networks with nonactive claims in case of an arbitrary distribution of servicing durations. It has been established that the stationary distribution of state probabilities in such networks has multiplicative form and is insensitive with respect to the functional form of the servicing duration distribution.

In [7], V.A. Ivnitskii in his studies of non-Markov queueing networks introduced the notion of piecewise linear (PLQN) and piecewise continuous queueing networks (PCQN). Servicing in such networks has "energy-based" rather than "time-based" meaning, i.e., each servicing operation is characterized by a random value of the work that needs to be done. Since for arbitrary distribution functions on the amount of work needed to service a claim the random process characterizing the number of claims in each node will not be Markov, that work, similar to most works on insensitivity, uses the method of extending the phase space (with additional variables). Depending on how the additional variables characterizing the residual amount of work needed to finish a servicing operation behave, the non-Markov networks are divided into PLQN and PCQN. For PLQN, additional variables $\xi(t)$ decrease in a linear fashion, and the rate of decrease may depend on the node state or the network state as a whole,

$$
\frac{d \xi(t)}{d t}=-\alpha
$$

For PCQN, the rate of decrease depends on the residual amount of work. This dependence is given by some continuous function

$$
\frac{d \xi(t)}{d t}=-\beta(\xi(t))
$$

The work [7] shows insensitivity results for many open and closed queueing networks with different "instantaneous" disciplines: for a Jackson network where servicing and circulation parameters depend on the network state, for a network with different classes of claims and servicing and circulation flow parameters depending on the network state, for a network with generalized group servicing, a network with deterministic circulation, for a closed star-like network, an open network with losses, and many other queueing networks.

The energy-based interpretation generalizes the notion of a servicing process, is highly interesting from the practical point of view, and lets one consider a much wider class of problems and study more complex and more interesting network models. For instance, the works $[6,10,11]$ find the form of the stationary distribution of state probabilities, ergodicity conditions, and establish the insensitivity of the stationary distribution of state probabilities with respect to the functional form of the distribution of the amount of work needed to service claims. Networks with negative claims and multimodal strategies are considered in [12], which establishes the insensitivity of stationary distribution state probabilities with respect to the functional form of the distribution of amount of work needed to switch modes. The work [13] establishes the insensitivity of the stationary distribution in case of energy-based setting for a closed queueing network with non-active customers.

In this work, we consider a generalization of the model from [8] to the case of the "energy-based" interpretation of the servicing process. The purpose of this work is to study an open PLQN with temporarily non-active claims and informational signals. We assume that amounts of work needed to service claims at the nodes are distributed according to an arbitrary law. We establish the insensitivity of the stationary distribution of network states with respect to the functional form of the distribution of the amounts of work needed to service claims for fixed first moments.

\section{NETWORK MODEL DESCRIPTION}

We consider an open queueing network with the set of nodes $J=\{1, \ldots, N\}$. Nodes of the network receive from the outside independent Poisson flows of claims with intensities $\lambda_{i}, i \in J$. All 
claims in the network are divided into regular, which can be serviced, and inactive. We assume that nodes of the network receive from the outside independent Poisson flows of informational signals with intensities $\nu_{i}$ and $\varphi_{i}, i \in J$. An information signal arrived to the $i$ th node with intensity $\nu_{i}$ reduces the number of regular claims by one and increases by one the number of nonactive claims; in case when there are no regular claims at the $i$ th node the signal leaves the network. An informational signal arriving at the $i$ th node with intensity $\varphi_{i}$ reduces the number of non-active claims by one and increases by one the number of regular claims; if there are no non-active claims at the $i$ th node the signal leaves the network. Informational signals do not require servicing.

The network state at time moment $t$ is characterized by a vector $z(t)=\left(\left(n_{i}(t), n_{i}^{\prime}(t)\right), i \in J\right)$, where $\left(n_{i}(t), n_{i}^{\prime}(t)\right)$ is the state of the $i$ th node at time moment $t$. Here $n_{i}(t)$ and $n_{i}^{\prime}(t)$ are the number of regular and non-active claims respectively at the $i$ th node at time moment $t$, and the total number of claims at the $i$ th node is $n_{i}(t)+n_{i}^{\prime}(t)$. The process $z(t)$ has countable phase space $Z$.

We number regular claims in each node's queue from the "tail" of the queue to the servicing device, i.e., if the $i$ th node has $n_{i}$ regular (active) claims, then the claim currently being serviced is numbered $n_{i}$, and the last claim in the queue is numbered 1 . Inactive claims in the $i$ th node's queue are numbered as follows: the claim that last became inactive has number $n_{i}^{\prime}$. A signal $\nu_{i}$ arriving at node $i$ acts on a regular claim numbered 1 , which becomes an inactive claim number $n_{i}^{\prime}+1$. A signal $\varphi_{i}$ acts on a non-active claim with number $n_{i}^{\prime}$, which becomes a regular claim number 1 .

The servicing discipline is $L C F S-P R$. A claim arriving at node $i$ begins servicing immediately and is assigned number $n_{i}+1$, and a pushed out claim keeps number $n_{i}$ and becomes the first in the afterservice queue. We assume that at the initial time moment there are no temporarily non-active claims in the network.

If at time moment $t$ the state of the $i$ th node is a vector $\left(n_{i}(t), n_{i}^{\prime}(t)\right)$, and immediately after this moment the node receives a claim, which, as we have noted above, starts servicing immediately, then the amount of work for servicing it becomes a random value $n_{i}\left(n_{i}+n_{i}^{\prime}+1\right)$ with distribution function $B_{i}\left(n_{i}+n_{i}^{\prime}+1, z\right)$ and mean $\tau_{i}\left(n_{i}+n_{i}^{\prime}+1\right)<\infty$. We assume that $B_{i}\left(n_{i}+n_{i}^{\prime}+1,0\right)=0, i \in J$. If at time moment $t$ the state of the $i$ th node is $\left(n_{i}(t), n_{i}^{\prime}(t)\right)$, servicing is done with rate $\alpha_{i}\left(n_{i}+n_{i}^{\prime}\right)$, i.e., it depends on the node state, $i \in J$. Servicing in such network has "energy-based" rather than "time-based" interpretation: each servicing operation is characterized by a random value of the work that has to be done. A claim that has been serviced at the $i$ th node instantaneously with probability $p_{i, j}$ passes to node $j$, and with probability $p_{i, 0}$ leaves the network $\left(\sum_{j \in J} p_{i, j}+p_{i, 0}=1, i \in J\right)$. Without loss of generality we assume that $p_{i, i}=0, i \in J$. The routing matrix is assumed to be irreducible.

It has been shown for open networks that if the routing matrix $\left(p_{i, j}\right)$ is irreducible the system of traffic equations

$$
\varepsilon_{j}=\lambda_{j}+\sum_{i=1}^{N} \varepsilon_{i} p_{i, j}, \quad j \in J,
$$

has a unique positive solution $\left\{\varepsilon_{j}, j \in J\right\}[4]$.

\section{INSENSITIVITY OF THE STATIONARY PROBABILITY DISTRIBUTION FOR NETWORK STATES}

The work [1] considers the case when $B_{i}\left(n_{i}+n_{i}^{\prime}, z\right)=1-\exp \left\{-\mu_{i} z\right\}\left(\mu_{i}>0, z>0\right), \tau_{i}\left(n_{i}+n_{i}^{\prime}\right)=$ $1 / \mu_{i}$ with unit servicing rate $\alpha_{i}\left(n_{i}+n_{i}^{\prime}\right)=1$, i.e., in this case $B_{i}\left(n_{i}+n_{i}^{\prime}, z\right)$ is the distribution function of the exponential servicing time, $i \in J$. Then $z(t)$ is a Markov process that satisfies the following theorem. 
Theorem 1 [1]. Given that

$$
\begin{aligned}
\varepsilon_{i} & <\mu_{i}, \\
\varepsilon_{i} \nu_{i} & <\mu_{i} \varphi_{i}
\end{aligned}
$$

the Markov process $z(t)$ is ergodic, and the stationary distribution of state probabilities for this process is

$$
p\left(\left(n_{1}, n_{1}^{\prime}\right), \ldots,\left(n_{N}, n_{N}^{\prime}\right)\right)=p_{1}\left(n_{1}, n_{1}^{\prime}\right) \ldots p_{N}\left(n_{N}, n_{N}^{\prime}\right) .
$$

Here $\left(\left(n_{1}, n_{1}^{\prime}\right), \ldots,\left(n_{N}, n_{N}^{\prime}\right)\right) \in Z$, and

$$
p_{i}\left(n_{i}, n_{i}^{\prime}\right)=\left(1-\frac{\varepsilon_{i}}{\mu_{i}}\right)\left(\frac{\varepsilon_{i}}{\mu_{i}}\right)^{n_{i}}\left(1-\frac{\varepsilon_{i} \nu_{i}}{\mu_{i} \varphi_{i}}\right)\left(\frac{\varepsilon_{i} \nu_{i}}{\mu_{i} \varphi_{i}}\right)^{n_{i}^{\prime}}
$$

is the stationary distribution of state probabilities at the $i$ th node, $\left\{\varepsilon_{j}, j \in J\right\}$ is a solution of the system of traffic Eqs. (1).

The work [9] has considered a generalization of the model from [1] to the case of an arbitrary distribution of servicing durations. Stationary distribution of state probabilities and ergodicity conditions have been found, and it has been proven that of the stationary distribution of state probabilities is insensitive with respect to the functional form of the servicing durations distribution. In [8], a similar result has been obtained for the case of a closed network with non-active claims.

We now turn to a more general case, considering the energy-based interpretation of the servicing process. Suppose that the amount of work needed to service a claim is a random value $\eta_{i}\left(n_{i}+n_{i}^{\prime}\right)$ with an arbitrary distribution function $B_{i}\left(n_{i}+n_{i}^{\prime}, z\right)$ and expectation $\tau_{i}\left(n_{i}+n_{i}^{\prime}\right)<\infty$. Let $\psi_{i, k}(t)$ denote the amount of work remaining to be done from time moment $t$ to finish servicing the claim which at time moment $t$ occupies the $k$ th position at the $i$ th node, $\psi_{i}(t)=\left(\psi_{i, 1}(t), \ldots, \psi_{i, n_{i}+n_{i}^{\prime}}(t)\right)$, $i \in J$. Due to the above, if the state of the $i$ th node is $\left(n_{i}, n_{i}^{\prime}\right)$ then

$$
\frac{d \psi_{i, n_{i}+n_{i}^{\prime}}(t)}{d t}=-\alpha_{i}\left(n_{i}+n_{i}^{\prime}\right), \quad i \in J
$$

Then in the general case the process $z(t)$ is not Markov, and we can consider a piecewise linear Markov process $\zeta(t)=(z(t), \psi(t))$ by adding to $z(t)$ a continuous component $\psi(t)=\left(\psi_{1}(t), \ldots\right.$, $\left.\psi_{N}(t)\right)$.

We introduce the following notation:

$$
\begin{gathered}
F(z, x)=F\left(z, x_{1,1}, \ldots, x_{1, n_{1}+n_{1}^{\prime}} ; x_{2,1}, \ldots, x_{2, n_{2}+n_{2}^{\prime}} ; \ldots ; x_{N, 1}, \ldots, x_{N, n_{N}+n_{N}^{\prime}}\right) \\
=\lim _{t \rightarrow \infty} P\left\{z(t)=z, \psi_{i, 1}(t)<x_{i, 1}, \ldots, \psi_{i, n_{i}+n_{i}^{\prime}}(t)<x_{i, n_{i}+n_{i}^{\prime}}, i \in J\right\} \\
z \in Z, x_{k, l} \in \mathbb{R} \forall k, l .
\end{gathered}
$$

Functions $F(z, x)$ are called stationary distribution functions of state probabilities for the piecewise linear process $\zeta(t)$, since for every fixed $z$ function $F(z, x)$ in the part of continuous components is a distribution function.

Theorem 2. If it holds that

$$
\sum_{z \in Z} q(z) \prod_{i=1}^{N}\left(\varepsilon_{i}^{n_{i}}\left(\frac{\varepsilon_{i} \nu_{i}}{\varphi_{i}}\right)^{n_{i}^{\prime}} \prod_{s=1}^{n_{i}+n_{i}^{\prime}} \tau_{i}(s) \alpha_{i}(s)^{-1}\right)<\infty
$$


where

$$
q(z)=\sum_{i=1}^{N}\left(\lambda_{i}+\tau_{i}(s)^{-1} \alpha_{i}(s)+\nu_{i}+\varphi_{i}\right),
$$

the process $\zeta(t)$ is ergodic, and the stationary distributions of functions of state probabilities $F(z, x)$ are given by formulas

$$
\begin{aligned}
& F(z, x)=p_{1}\left(n_{1}, n_{1}^{\prime}\right) p_{2}\left(n_{2}, n_{2}^{\prime}\right) \ldots p_{N}\left(n_{N}, n_{N}^{\prime}\right) \\
& \times \prod_{i=1}^{N} \prod_{s=1}^{n_{i}+n_{i}^{\prime}} \frac{1}{\tau_{i}(s)} \int_{0}^{x_{i, s}}\left(1-B_{i}(s, u)\right) d u, z \in Z
\end{aligned}
$$

where

$$
p_{i}\left(n_{i}, n_{i}^{\prime}\right)=\varepsilon_{i}^{n_{i}}\left(\frac{\varepsilon_{i} \nu_{i}}{\varphi_{i}}\right)^{n_{i}^{\prime}} \prod_{s=1}^{n_{i}+n_{i}^{\prime}} \frac{\tau_{i}(s)}{\alpha_{i}(s)} p_{i}(0,0),
$$

$\varepsilon_{i}$ can be found from (1), and

$$
p_{i}(0,0)=\left(\sum_{n_{i}=0}^{\infty} \sum_{n_{i}^{\prime}=0}^{\infty} \varepsilon_{i}^{n_{i}}\left(\frac{\varepsilon_{i} \nu_{i}}{\varphi_{i}}\right)^{n_{i}^{\prime}} \prod_{s=1}^{n_{i}+n_{i}^{\prime}} \frac{\tau_{i}(s)}{\alpha_{i}(s)}\right)^{-1}, \quad i \in J
$$

Proof of Theorem 2 is given in the Appendix.

We denote by $\{p(z), z \in Z\}$ the stationary distribution of state probabilities for the process $z(t)$. Theorem 2 together with equality $p(z)=F(z,+\infty)$ implies the following corollary.

Corollary. If relation (5) holds then process $z(t)$ is ergodic, and its stationary distribution of state probabilities $\{p(z), z \in Z\}$ does not depend on the functional form of the distributions $B_{i}(s, x)$, $i \in J$, and has multiplicative form

$$
p(z)=p_{1}\left(n_{1}, n_{1}^{\prime}\right) p_{2}\left(n_{2}, n_{2}^{\prime}\right) \ldots p_{N}\left(n_{N}, n_{N}^{\prime}\right)
$$

where $p_{i}\left(n_{i}, n_{i}^{\prime}\right)$ are given by formulas $(7),(8)$. Here $p_{i}\left(n_{i}, n_{i}^{\prime}\right), i \in J$, is the stationary distribution of state probabilities at the isolated node.

\section{CONCLUSION}

We have studied the operation of an open queueing network with non-active customers and informational signals. The servicing discipline was an absolute priority of incoming claims with afterserviced of claims pushed out of a servicing device. For the case of an arbitrary distribution of the amount of work needed to service a claim we have established that the stationary distribution of network states is insensitive with respect to the functional form of the distributions of the amounts of work for fixed first moments. We have found an ergodicity condition. It has been established that the stationary distribution of the network has the form of a product where each factor is a distribution at a separate node in a fictional environment. The results of our studies of these networks can be used in practice to analyze stationary operation of real objects that have network structure and admit non-active state of claims. 


\section{ACKNOWLEDGMENTS}

This work was supported by the Belarus Republican Foundation for Basic Research, project no. f14m-099.

APPENDIX

Proof of Theorem 2. Consider the process $\zeta(t)$. Under condition $(5) \zeta(t)$ is ergodic. A formal proof of this fact can proceed with Smith's limit theorem [10] if we take into account that random process $\zeta(t)$ is regenerating. Indeed, the network's operation can be schematically represented as an alternation of periods when the network is at the " 0 " state (the nodes have neither regular nor non-active claims) and periods when the network is busy (otherwise). The moment when the network transitions into the free state " 0 " is the moment of regeneration, and then the proof reduces to applying Smith's theorem for regenerating processes.

We call changes in the state of the piecewise linear process $\zeta(t)$ due to claims arriving in the network or informational signals that transfer claims from regular state to non-active and vice versa spontaneous changes.

We denote by $e_{i} \in Z$ the vector all of whose coordinates are zero except for $\left(n_{i}, n_{i}^{\prime}\right)=(1,0)$; by $e_{i}^{\prime} \in Z$, the vector all of whose coordinates are zero except $\left(n_{i}, n_{i}^{\prime}\right)=(0,1)$.

Let $h$ be small. Consider the probability of the event

$$
P\left\{z(t+h)=z, \psi_{i, 1}(t+h)<x_{i, 1}, \ldots, \psi_{i, n_{i}+n_{i}^{\prime}}(t+h)<x_{i, n_{i}+n_{i}^{\prime}}, i \in J\right\} .
$$

This event can occur in the following mutually exclusive ways.

1. Since time moment $t$ over time $h$ there have been no spontaneous changes, and servicing has not finished in any node. The probability of this case is

$$
\begin{gathered}
P\left\{z(t)=z, \psi_{i, 1}(t)<x_{i, 1}, \ldots, \alpha_{i}\left(n_{i}+n_{i}^{\prime}\right) h I_{n_{i}>0} \leqslant \psi_{i, n_{i}+n_{i}^{\prime}}(t)\right. \\
\left.<x_{i, n_{i}+n_{i}^{\prime}}+\alpha_{i}\left(n_{i}+n_{i}^{\prime}\right) h I_{n_{i}>0}, i \in J\right\} \\
\times\left(1-\sum_{i=1}^{N}\left(\lambda_{i}+\nu_{i} I_{n_{i}>0}+\varphi_{i} I_{n_{i}^{\prime}>0}\right) h+o(h)\right) .
\end{gathered}
$$

2. Over time $h$, a claim has arrived at the $i$ th node which started servicing immediately, no node has finished servicing, and there have been no other spontaneous changes. The probability of this case is

$$
\begin{aligned}
& P\{z(t)= z-e_{i}, \psi_{k, 1}(t)<x_{k, 1}, \ldots, \alpha_{k}\left(n_{k}+n_{k}^{\prime}\right) h I_{n_{k}>0} \leqslant \psi_{k, n_{k}+n_{k}^{\prime}}(t) \\
&<x_{k, n_{k}+n_{k}^{\prime}}+\alpha_{k}\left(n_{k}+n_{k}^{\prime}\right) h I_{n_{k}>0}, k \in J, \quad k \neq i, \\
& \psi_{i, 1}(t)<x_{i, 1}, \ldots, \alpha_{i}\left(n_{i}+n_{i}^{\prime}-1\right)(h-\theta) I_{n_{i}>1} \leqslant \psi_{i, n_{i}+n_{i}^{\prime}-1}(t) \\
&<\left.x_{i, n_{i}+n_{i}^{\prime}-1}+\alpha_{i}\left(n_{i}+n_{i}^{\prime}-1\right)(h-\theta) I_{n_{i}>1}, i \in J\right\} \\
& \times B_{i}\left(n_{i}+n_{i}^{\prime}, x_{i, n_{i}+n_{i}^{\prime}}+\alpha_{i}\left(n_{i}+n_{i}^{\prime}\right) \theta\right) I_{n_{i}>0}\left(\lambda_{i} h+o(h)\right)
\end{aligned}
$$

where $(h-\theta)$ is the time that has passed since time moment $t$ until the claim arrived, and $\theta$ is the time from the moment when the claim arrived to $t+h, 0<\theta<h$. 
3. Over time $h$, a claim has been serviced at the $j$ th node and immediately transferred to the $i$ th node, and there have been no spontaneous changes. The probability of this event is

$$
\begin{gathered}
P\left\{z(t)=z-e_{i}+e_{j}, \psi_{k, 1}(t)<x_{k, 1}, \ldots, \alpha_{k}\left(n_{k}+n_{k}^{\prime}\right) h I_{n_{k}>0} \leqslant \psi_{k, n_{k}+n_{k}^{\prime}}(t)\right. \\
<x_{k, n_{k}+n_{k}^{\prime}}+\alpha_{k}\left(n_{k}+n_{k}^{\prime}\right) h I_{n_{k}>0}, k \in J, k \neq i, k \neq j \\
\psi_{j, 1}(t)<x_{j, 1}, \ldots, \psi_{j, n_{j}+n_{j}^{\prime}}(t)<x_{j, n_{j}+n_{j}^{\prime}}, \psi_{j, n_{j}+n_{j}^{\prime}+1}(t) \\
<\alpha_{j}\left(n_{j}+n_{j}^{\prime}+1\right)(h-\theta) \\
\psi_{i, 1}(t)<x_{i, 1}, \ldots, \alpha_{i}\left(n_{i}+n_{i}^{\prime}-1\right)(h-\theta) I_{n_{i}>1} \leqslant \psi_{i, n_{i}+n_{i}^{\prime}-1}(t) \\
\left.<x_{i, n_{i}+n_{i}^{\prime}-1}+\alpha_{i}\left(n_{i}+n_{i}^{\prime}-1\right)(h-\theta) I_{n_{i}>1}\right\} \\
\times B_{i}\left(n_{i}+n_{i}^{\prime}, x_{i, n_{i}+n_{i}^{\prime}}+\alpha_{i}\left(n_{i}+n_{i}^{\prime}\right) \theta\right) p_{j, i} I_{n_{i}>0}+o(h)
\end{gathered}
$$

where $(h-\theta)$ is the time that passed since time moment $t$ until the claim finished servicing, $0<\theta<h$.

4. Over time $h$, a claim has been serviced at the $j$ th node and then left the network, and there have been no spontaneous changes. The probability of this event is

$$
\begin{gathered}
P\left\{z(t)=z+e_{j}, \psi_{k, 1}(t)<x_{k, 1}, \ldots, \alpha_{k}\left(n_{k}+n_{k}^{\prime}\right) h I_{n_{k}>0} \leqslant \psi_{k, n_{k}+n_{k}^{\prime}}(t)\right. \\
<x_{k, n_{k}+n_{k}^{\prime}}+\alpha_{k}\left(n_{k}+n_{k}^{\prime}\right) h I_{n_{k}>0}, k \in J, k \neq j \\
\psi_{j, 1}(t)<x_{j, 1}, \ldots, \psi_{j, n_{j}+n_{j}^{\prime}}(t)<x_{j, n_{j}+n_{j}^{\prime}}, \psi_{j, n_{j}+n_{j}^{\prime}+1}(t) \\
\left.<\alpha_{j}\left(n_{j}+n_{j}^{\prime}+1\right)(h-\theta)\right\} p_{j, 0}+o(h) .
\end{gathered}
$$

5. Over time $h$, an informational signal of intensity $\nu_{i}$ has arrived at the $j$ th node, and it decreased the number of regular claims by one and increased by one the number of non-active claims; there have been no other spontaneous changes, servicing has not ended at any node. The probability of this case is

$$
\begin{gathered}
P\left\{z(t)=z+e_{i}-e_{i}^{\prime}, \psi_{k, 1}(t)<x_{k, 1}, \ldots, \alpha_{k}\left(n_{k}+n_{k}^{\prime}\right) h I_{n_{k}>0} \leqslant \psi_{k, n_{k}+n_{k}^{\prime}}(t)\right. \\
<x_{k, n_{k}+n_{k}^{\prime}}+\alpha_{k}\left(n_{k}+n_{k}^{\prime}\right) h I_{n_{k}>0}, k \in J, k \neq i \\
\psi_{i, 1}(t)<x_{i, 1}, \ldots, \alpha_{i}\left(n_{i}+n_{i}^{\prime}\right) h \leqslant \psi_{i, n_{i}+n_{i}^{\prime}}(t) \\
\left.<x_{i, n_{i}+n_{i}^{\prime}}+\alpha_{i}\left(n_{i}+n_{i}^{\prime}\right) h\right\}\left(\nu_{i} h+o(h)\right) I_{n_{i}^{\prime}>0} .
\end{gathered}
$$

6. Over time $h$, an informational signal of intensity $\varphi_{i}$ has arrived at the $j$ th node, and it decreased the number of non-active claims by one and increased by one the number of regular claims, there have been no other spontaneous changes, and servicing has not finished at any node. The probability of this event is

$$
\begin{gathered}
P\left\{z(t)=z-e_{i}+e_{i}^{\prime}, \psi_{k, 1}(t)<x_{k, 1}, \ldots, \alpha_{k}\left(n_{k}+n_{k}^{\prime}\right) h I_{n_{k}>0} \leqslant \psi_{k, n_{k}+n_{k}^{\prime}}(t)\right. \\
<x_{k, n_{k}+n_{k}^{\prime}}+\alpha_{k}\left(n_{k}+n_{k}^{\prime}\right) h I_{n_{k}>0}, k \in J, k \neq i \\
\psi_{i, 1}(t)<x_{i, 1}, \ldots, \alpha_{i}\left(n_{i}+n_{i}^{\prime}\right) h I_{n_{i}>1} \leqslant \psi_{i, n_{i}+n_{i}^{\prime}}(t) \\
\left.<x_{i, n_{i}+n_{i}^{\prime}}+\alpha_{i}\left(n_{i}+n_{i}^{\prime}\right) h I_{n_{i}>1}\right\}\left(\varphi_{i} h+o(h)\right) I_{n_{i}>0}
\end{gathered}
$$

7. Finally, over time $h$ there have been at least two changes in the network state. The probability of this event is $o(h)$. 
Naturally, each of these cases and each node has its own parameter $\theta$, but we do not introduce any indexing for $\theta$ in order to make computations less tedious.

Due to the above we have that

$$
\begin{aligned}
& P\left\{z(t+h)=z, \psi_{i, 1}(t+h)<x_{i, 1}, \ldots, \psi_{i, n_{i}+n_{i}^{\prime}}(t+h)<x_{i, n_{i}+n_{i}^{\prime}}, i \in J\right\} \\
& =P\left\{z(t)=z, \psi_{i, 1}(t)<x_{i, 1}, \ldots, \alpha_{i}\left(n_{i}+n_{i}^{\prime}\right) h I_{n_{i}>0} \leqslant \psi_{i, n_{i}+n_{i}^{\prime}}(t)\right. \\
& \left.<x_{i, n_{i}+n_{i}^{\prime}}+\alpha_{i}\left(n_{i}+n_{i}^{\prime}\right) h I_{n_{i}>0}, i \in J\right\}\left(1-\sum_{i=1}^{N}\left(\lambda_{i}+\nu_{i} I_{n_{i}>0}+\varphi_{i} I_{n_{i}^{\prime}>0}\right) h+o(h)\right) \\
& +\sum_{i=1}^{N} P\left\{z(t)=z-e_{i}, \psi_{k, 1}(t)<x_{k, 1}, \ldots, \alpha_{k}\left(n_{k}+n_{k}^{\prime}\right) h I_{n_{k}>0} \leqslant \psi_{k, n_{k}+n_{k}^{\prime}}(t)\right. \\
& <x_{k, n_{k}+n_{k}^{\prime}}+\alpha_{k}\left(n_{k}+n_{k}^{\prime}\right) h I_{n_{k}>0}, k \in J, k \neq i, \\
& \psi_{i, 1}(t)<x_{i, 1}, \ldots, \alpha_{i}\left(n_{i}+n_{i}^{\prime}-1\right)(h-\theta) I_{n_{i}>1} \leqslant \psi_{i, n_{i}+n_{i}^{\prime}-1}(t) \\
& \left.<x_{i, n_{i}+n_{i}^{\prime}-1}+\alpha_{i}\left(n_{i}+n_{i}^{\prime}-1\right)(h-\theta) I_{n_{i}>1}, i \in J\right\} \\
& \times B_{i}\left(n_{i}+n_{i}^{\prime}, x_{i, n_{i}+n_{i}^{\prime}}+\alpha_{i}\left(n_{i}+n_{i}^{\prime}\right) \theta\right) I_{n_{i}>0}\left(\lambda_{i} h+o(h)\right) \\
& +\sum_{i=1}^{N} \sum_{j=1, j \neq i}^{N} P\left\{z(t)=z-e_{i}+e_{j}, \psi_{k, 1}(t)<x_{k, 1}, \ldots, \alpha_{k}\left(n_{k}+n_{k}^{\prime}\right) h I_{n_{k}>0}\right. \\
& \leqslant \psi_{k, n_{k}+n_{k}^{\prime}}(t)<x_{k, n_{k}+n_{k}^{\prime}}+\alpha_{k}\left(n_{k}+n_{k}^{\prime}\right) h I_{n_{k}>0}, k \in J, k \neq i, k \neq j, \\
& \psi_{j, 1}(t)<x_{j, 1}, \ldots, \psi_{j, n_{j}+n_{j}^{\prime}}(t)<x_{j, n_{j}+n_{j}^{\prime}}, \psi_{j, n_{j}+n_{j}^{\prime}+1}(t)<\alpha_{j}\left(n_{j}+n_{j}^{\prime}+1\right)(h-\theta), \\
& \psi_{i, 1}(t)<x_{i, 1}, \ldots, \alpha_{i}\left(n_{i}+n_{i}^{\prime}-1\right)(h-\theta) I_{n_{i}>1} \leqslant \psi_{i, n_{i}+n_{i}^{\prime}-1}(t) \\
& \left.<x_{i, n_{i}+n_{i}^{\prime}-1}+\alpha_{i}\left(n_{i}+n_{i}^{\prime}-1\right)(h-\theta) I_{n_{i}>1}\right\} \\
& \times B_{i}\left(n_{i}+n_{i}^{\prime}, x_{i, n_{i}+n_{i}^{\prime}}+\alpha_{i}\left(n_{i}+n_{i}^{\prime}\right) \theta\right) p_{j, i} I_{n_{i}>0}+o(h) \\
& +\sum_{j=1}^{N} P\left\{z(t)=z+e_{j}, \psi_{k, 1}(t)<x_{k, 1}, \ldots, \alpha_{k}\left(n_{k}+n_{k}^{\prime}\right) h I_{n_{k}>0} \leqslant \psi_{k, n_{k}+n_{k}^{\prime}}(t)\right. \\
& <x_{k, n_{k}+n_{k}^{\prime}}+\alpha_{k}\left(n_{k}+n_{k}^{\prime}\right) h I_{n_{k}>0}, k \in J, k \neq j \\
& \left.\psi_{j, 1}(t)<x_{j, 1}, \ldots, \psi_{j, n_{j}+n_{j}^{\prime}}(t)<x_{j, n_{j}+n_{j}^{\prime}}, \psi_{j, n_{j}+n_{j}^{\prime}+1}(t)<\alpha_{j}\left(n_{j}+n_{j}^{\prime}+1\right)(h-\theta)\right\} p_{j, 0}+o(h) \\
& +\sum_{i=1}^{N} P\left\{z(t)=z+e_{i}-e_{i}^{\prime}, \psi_{k, 1}(t)<x_{k, 1}, \ldots, \alpha_{k}\left(n_{k}+n_{k}^{\prime}\right) h I_{n_{k}>0}\right. \\
& \leqslant \psi_{k, n_{k}+n_{k}^{\prime}}(t)<x_{k, n_{k}+n_{k}^{\prime}}+\alpha_{k}\left(n_{k}+n_{k}^{\prime}\right) h I_{n_{k}>0}, k \in J, k \neq i \\
& \left.\psi_{i, 1}(t)<x_{i, 1}, \ldots, \alpha_{i}\left(n_{i}+n_{i}^{\prime}\right) h \leqslant \psi_{i, n_{i}+n_{i}^{\prime}}(t)<x_{i, n_{i}+n_{i}^{\prime}}+\alpha_{i}\left(n_{i}+n_{i}^{\prime}\right) h\right\}\left(\nu_{i} h+o(h)\right) I_{n_{i}^{\prime}>0} \\
& +\sum_{i=1}^{N} P\left\{z(t)=z-e_{i}+e_{i}^{\prime}, \psi_{k, 1}(t)<x_{k, 1}, \ldots, \alpha_{k}\left(n_{k}+n_{k}^{\prime}\right) h I_{n_{k}>0}\right. \\
& \leqslant \psi_{k, n_{k}+n_{k}^{\prime}}(t)<x_{k, n_{k}+n_{k}^{\prime}}+\alpha_{k}\left(n_{k}+n_{k}^{\prime}\right) h I_{n_{k}>0}, k \in J, k \neq i, \\
& \psi_{i, 1}(t)<x_{i, 1}, \ldots, \alpha_{i}\left(n_{i}+n_{i}^{\prime}\right) h I_{n_{i}>1} \leqslant \psi_{i, n_{i}+n_{i}^{\prime}}(t) \\
& \left.<x_{i, n_{i}+n_{i}^{\prime}}+\alpha_{i}\left(n_{i}+n_{i}^{\prime}\right) h I_{n_{i}>1}\right\}\left(\varphi_{i} h+o(h)\right) I_{n_{i}>0}+o(h) .
\end{aligned}
$$


Further, let us express each probability in the equations above via functions of the form

$$
F_{t}(z, x)=P\left\{z(t)=z, \psi_{i, 1}(t)<x_{i, 1}, \ldots, \psi_{i, n_{i}+n_{i}^{\prime}}(t)<x_{i, n_{i}+n_{i}^{\prime}}, i \in J\right\} .
$$

Considering $F_{t}(z, x)$ as complex functions of $h$ and assuming that they have first order partial derivatives with respect to variables $t$ and $x_{i, n_{i}+n_{i}^{\prime}}$, we write decompositions of these functions in Taylor series with residual term in the form of Peano, taking into account that

$$
\begin{gathered}
P\left\{z(t)=z, \psi_{i, 1}(t)<x_{i, 1}, \ldots, \alpha_{i}\left(n_{i}+n_{i}^{\prime}\right) h \leqslant \psi_{i, n_{i}+n_{i}^{\prime}}(t)<x_{i, n_{i}+n_{i}^{\prime}}+\alpha_{i}\left(n_{i}+n_{i}^{\prime}\right) h, i \in J\right\} \\
=F_{t}\left(z, x_{i, 1}, \ldots, x_{i, n_{i}+n_{i}^{\prime}}+\alpha_{i}\left(n_{i}+n_{i}^{\prime}\right) h, i \in J\right) \\
-\sum_{k=1}^{N} F_{t}\left(z, x_{i, 1}, \ldots, x_{i, n_{i}+n_{i}^{\prime}}+\alpha_{i}\left(n_{i}+n_{i}^{\prime}\right) h, i \in J, i \neq k ; x_{k, 1}, \ldots, x_{k, n_{k}+n_{k}^{\prime}-1}, \alpha_{k}\left(n_{k}+n_{k}^{\prime}\right) h\right)+\ldots \\
+F_{t}\left(z, x_{i, 1}, \ldots, x_{i, n_{i}+n_{i}^{\prime}-1}, \alpha_{i}\left(n_{i}+n_{i}^{\prime}\right) h, i \in J\right) .
\end{gathered}
$$

Obviously, those functions $F_{t}(z, x)$ that have $h$ as their arguments at least twice will give $o(h)$ in their Taylor series decompositions. Therefore,

$$
\begin{gathered}
P\left\{z(t)=z, \psi_{i, 1}(t)<x_{i, 1}, \ldots, \alpha_{i}\left(n_{i}+n_{i}^{\prime}\right) h \leqslant \psi_{i, n_{i}+n_{i}^{\prime}}(t)<x_{i, n_{i}+n_{i}^{\prime}}+\alpha_{i}\left(n_{i}+n_{i}^{\prime}\right) h, i \in J\right\} \\
=F_{t}\left(z, x_{i, 1}, \ldots, x_{i, n_{i}+n_{i}^{\prime}}, i \in J\right)+\sum_{i=1}^{N} \frac{\partial F_{t}\left(z, x_{i, 1}, \ldots, x_{i, n_{i}+n_{i}^{\prime}}, i \in J\right)}{\partial x_{i, n_{i}+n_{i}^{\prime}}} \alpha_{i}\left(n_{i}+n_{i}^{\prime}\right) h \\
-\sum_{i=1}^{N} \frac{\partial F_{t}\left(z, x_{l, 1}, \ldots, x_{l, n_{l}+n_{i}^{\prime}}, l \in J, l \neq i ; x_{i, 1}, \ldots, x_{i, n_{i}+n_{i}^{\prime}-1}, 0\right)}{\partial x_{i, n_{i}+n_{i}^{\prime}}} \alpha_{i}\left(n_{i}+n_{i}^{\prime}\right) h+o(h) .
\end{gathered}
$$

We also decompose expressions of the form $B_{i}\left(n_{i}+n_{i}^{\prime}, x_{i, n_{i}+n_{i}^{\prime}}+\alpha_{i}\left(n_{i}+n_{i}^{\prime}\right) \theta\right)$ into Taylor series as a function of the variable $\theta$.

Tending $t$ to infinity and taking into account that in this case the partial derivative $F_{t}(z, x)$ with respect to variable $t$ tends to zero, we arrive at the following system of equations:

$$
\begin{gathered}
F(z, x)=F(z, x)+h \sum_{i=1}^{N} \alpha_{i}\left(n_{i}+n_{i}^{\prime}\right)\left(\frac{\partial F(z, x)}{\partial x_{i, n_{i}+n_{i}^{\prime}}}-\left(\frac{\partial F(z, x)}{\partial x_{i, n_{i}+n_{i}^{\prime}}}\right)_{x_{i, n_{i}+n_{i}^{\prime}}=0}\right) I_{n_{i}>0} \\
-\left(\sum_{i=1}^{N}\left(\lambda_{i}+\nu i I_{n_{i}>0}+\varphi_{i} I_{n_{i}^{\prime}>0}\right) h+o(h)\right) F(z, x) \\
+\sum_{i=1}^{N}\left(\lambda_{i} h+o(h)\right) B_{i}\left(n_{i}+n_{i}^{\prime}, x_{i, n_{i}+n_{i}^{\prime}}\right) F\left(z-e_{i}, x\right) \\
+h \sum_{j=1}^{N} \sum_{i=1, i \neq j}^{N} \alpha_{j}\left(n_{j}+n_{j}^{\prime}+1\right) p_{j, i} B_{i}\left(n_{i}+n_{i}^{\prime}, x_{i, n_{i}+n_{i}^{\prime}}\right)\left(\frac{\partial F\left(z+e_{j}-e_{i}, x\right)}{\partial x_{j, n_{j}+n_{j}^{\prime}+1}}\right)_{x_{j, n_{j}+n_{j}^{\prime}+1}=0} I_{n_{i}>0} \\
+h \sum_{j=1}^{N} \alpha_{j}\left(n_{j}+n_{j}^{\prime}+1\right) p_{j, 0}\left(\frac{\partial F\left(z+e_{j}, x\right)}{\partial x_{j, n_{j}+n_{j}^{\prime}+1}}\right)_{x_{j, n_{j}+n_{j}^{\prime}+1}=0} \\
+\sum_{i=1}^{N} F\left(z+e_{i}-e_{i}^{\prime}, x\right)\left(\nu_{i} h+o(h)\right) I_{n_{i}^{\prime}>0}+\sum_{i=1}^{N} F\left(z-e_{i}+e_{i}^{\prime}, x\right)\left(\varphi_{i} h+o(h)\right) I_{n_{i}>0}+o(h) .
\end{gathered}
$$


Next we subtract $F(z, x)$ from both sides, divided the remaining nonzero right-hand side by $h$, and tend $h$ to zero. Thus, $F(z, x)$ satisfies the following system of differential-difference equations:

$$
\begin{gathered}
F(z, x) \sum_{i=1}^{N}\left(\lambda_{i}+\nu_{i} I_{n_{i}>0}+\varphi_{i} I_{n_{i}^{\prime}>0}\right) \\
=\sum_{i=1}^{N} \alpha_{i}\left(n_{i}+n_{i}^{\prime}\right)\left(\frac{\partial F(z, x)}{\partial x_{i, n_{i}+n_{i}^{\prime}}}-\left(\frac{\partial F(z, x)}{\partial x_{i, n_{i}+n_{i}^{\prime}}}\right)_{x_{i, n_{i}+n_{i}^{\prime}}=0}\right) I_{n_{i}>0} \\
+\sum_{i=1}^{N} \lambda_{i} B_{i}\left(n_{i}+n_{i}^{\prime}, x_{i, n_{i}+n_{i}^{\prime}}\right) F\left(z-e_{i}, x\right) \\
+\sum_{j=1}^{N} \sum_{i=1, i \neq j}^{N} \alpha_{j}\left(n_{j}+n_{j}^{\prime}+1\right) p_{j, i} B_{i}\left(n_{i}+n_{i}^{\prime}, x_{i, n_{i}+n_{i}^{\prime}}\right) \\
\times\left(\frac{\partial F\left(z+e_{j}-e_{i}, x\right)}{\partial x_{j, n_{j}+n_{j}^{\prime}+1}}\right)_{x_{j, n_{j}+n_{j}^{\prime}+1}=0} I_{n_{i}>0} \\
+\sum_{j=1}^{N} \alpha_{j}\left(n_{j}+n_{j}^{\prime}+1\right) p_{j, 0}\left(\frac{\partial F\left(z+e_{j}, x\right)}{\partial x_{j, n_{j}+n_{j}^{\prime}+1}}\right)_{x_{j, n_{j}+n_{j}^{\prime}+1}=0} \\
+\sum_{i=1}^{N} F\left(z+e_{i}-e_{i}^{\prime}, x\right) \nu_{i} I_{n_{i}^{\prime}>0}+\sum_{i=1}^{N} F\left(z-e_{i}+e_{i}^{\prime}, x\right) \varphi_{i} I_{n_{i}>0}
\end{gathered}
$$

We divide this system of equations into local balance equations:

$$
\begin{gathered}
\sum_{i=1}^{N} \lambda_{i} F(z, x)=\sum_{j=1}^{N} \alpha_{j}\left(n_{j}+n_{j}^{\prime}+1\right) p_{j, 0}\left(\frac{\partial F\left(z+e_{j}, x\right)}{\partial x_{j, n_{j}+n_{j}^{\prime}+1}}\right)_{x_{j, n_{j}+n_{j}^{\prime}+1}=0}, \\
F(z, x)\left(\nu_{i} I_{n_{i}>0}+\varphi_{i} I_{n_{i}^{\prime}>0}\right)=F\left(z+e_{i}-e_{i}^{\prime}, x\right) \nu_{i} I_{n_{i}^{\prime}>0}+F\left(z-e_{i}+e_{i}^{\prime}, x\right) \varphi_{i} I_{n_{i}>0} \\
\alpha_{i}\left(n_{i}+n_{i}^{\prime}\right)\left(\left(\frac{\partial F(z, x)}{\partial x_{i, n_{i}+n_{i}^{\prime}}}\right)_{x_{i, n_{i}+n_{i}^{\prime}}=0}-\frac{\partial F(z, x)}{\partial x_{i, n_{i}+n_{i}^{\prime}}}\right) I_{n_{i}>0} \\
=\lambda_{i} B_{i}\left(n_{i}+n_{i}^{\prime}, x_{i, n_{i}+n_{i}^{\prime}}\right) F\left(z-e_{i}, x\right) \\
+\sum_{j=1, j=i}^{N} \alpha_{j}\left(n_{j}+n_{j}^{\prime}+1\right) p_{j, i} B_{i}\left(n_{i}+n_{i}^{\prime}, x_{i, n_{i}+n_{i}^{\prime}}\right) \\
\times\left(\frac{\partial F\left(z+e_{j}-e_{i}, x\right)}{\partial x_{j, n_{j}+n_{j}^{\prime}+1}}\right)_{x_{j, n_{j}+n_{j}^{\prime}+1}=0} I_{n_{i}>0} i \in J .
\end{gathered}
$$

Let us show that distribution functions of probabilities $F(z, x)$ given by formulas $(6)$ are solutions of the system of Eqs. (A.4)-(A.6), and therefore also the system of Eqs. (A.3).

If $n_{i}>0$, then, substituting (6) into Eq. (A.6), canceling similar terms, and dividing both parts of the resulting relation by $B_{i}\left(n_{i}+n_{i}^{\prime}, x_{i, n_{i}+n_{i}^{\prime}}\right) F\left(z-e_{i}, x\right)$ we get the traffic Eq. (1); if $n_{i}=0$ then (A.6) becomes an identity. Substituting (6) into (A.4), we get the corollary of traffic equations $1=\sum_{j=1}^{N} \varepsilon_{j} p_{j, 0}$. Finally, substituting (6) into (A.5) we get an identity. 


\section{REFERENCES}

1. Tsitsiashvili, G.Sh. and Osipova, M., Distributions in Stochastic Network Models, New York: Nova Publishers, 2008.

2. Malinkovsky, Yu. and Bojarovich, J., An Open Queueing Network with Partly Non-active Customers, Queues: Flows, Systems, Networks, Proc. 21th Int. Conf. Modern Probabilistic Methods for Analysis and Optimization of Information and Telecommunication Networks, Minsk: BSU, 2011, pp. 34-37.

3. Boyarovich, Yu.S., Stationary Distribution of a Network with Various Kinds of Claims and Unreliable Requirements, in Teoriya veroyatnostei, matematicheskaya statistika $i$ ikh prilozheniya, Minsk: BGU, 2009, vol. 2, pp. 14-20.

4. Jackson, J.R., Network of Waiting Lines, Oper. Res., 1957, no. 4, pp. 518-521.

5. Sevast'yanov, B.A., Limit Theorem for Markov Processes and Its Application to Telephone Systems with Faults, Teor. Veroyat. Primen., 1957, vol. 2, no. 1, pp. 106-116.

6. Starovoitov, A.N., Invariance of the Stationary State Distribution in Networks with Multimodal Servicing Strategies, Probl. Peredachi Inf., 2006, vol. 42, no. 4, pp. 121-128.

7. Ivnitskii, V.A., Teoriya setei massovogo obsluzhivaniya (Queueing Networks Theory), Moscow: Fizmatlit, 2004.

8. Boyarovich, Yu.S., The Stationary Distribution Invariance of States in a Closed Queueing Network with Temporarily Non-Active Customers, Autom. Remote Control, 2012, vol. 73, no. 10, pp. 1616-1623.

9. Bojarovich, J. and Malinkovsky, Yu.V., Stationary Distribution Invariance of an Open Queueing Network with Temporarily Non-Active Customers, Tomsk State Univ. J. Control Comput. Sci, 2012, no. 20, pp. $62-70$.

10. Starovoitov, A.N., Stationary Distribution Invariance for States of an Open Network with Multimodal Servicing Strategies, Izv. Gomel'sk. Gos. Univ. im. F. Skoriny, 2005, no. 5, pp. 169-171.

11. Starovoitov, A.N., On Invariance of Stationary State Distributions in an Open Network with Multimodal Servicing Strategies, Izv. Gomel'sk. Gos. Univ. im. F. Skoriny, 2006, no. 4, pp. 159-161.

12. Starovoitov, A.N., Networks with Multimodal Servicing, Negative Claims, and Arbitrary Sojourn Time in Modes, Izv. Gomel'skogo Gos. Univ. im. F. Skoriny, 2007, no. 6, pp. 193-198.

13. Bojarovich, J. and Dudovskaya, Y., Stationary Distribution Insensitivity of a Closed Queueing Network with Non-Active Customers, in Information Technologies and Mathematical Modelling, Ser. Communications in Computer and Information Science, Berlin: Springer-Verlag, 2014, vol. 487, pp. 50-58.

14. Gnedenko, B.V. and Kovalenko, I.N., Vvedenie v teoriyu massovogo obsluzhivaniya (Introduction to Queueing Theory), Moscow: KomKniga, 2005.

This paper was recommended for publication by A.I. Lyakhov, a member of the Editorial Board 